\title{
Impact of "non-lethal" tarsal clipping on bumble bees (Bombus vosnesenskii) may depend on queen stage and worker size
}

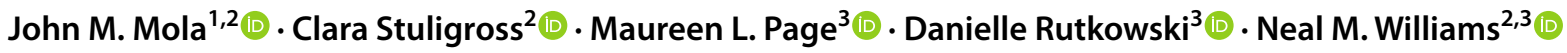

Received: 9 July 2020 / Accepted: 20 January 2021 / Published online: 8 February 2021

(C) This is a U.S. government work and not under copyright protection in the U.S.; foreign copyright protection may apply 2021

\begin{abstract}
Recent bumble bee declines have prompted the development of novel population monitoring tools, including the use of putatively non-lethal tarsal clipping to obtain genetic material. However, the potential side effects of tarsal clipping have only been tested in the worker caste of a single domesticated species, prompting the need to more broadly test whether tarsal clipping negatively affects sampled individuals. To determine if tarsal clipping reduces queen survivorship and colony establishment, we collected wild queens of Bombus vosnesenskii and clipped tarsi from a single leg of half the individuals. We reared captive queens and estimated survivorship and nest establishment success. We also clipped tarsi of workers from a subset of colonies across a range of body sizes. We found no consistent negative effect of clipping on queen survival. In the first year, clipped nest-searching queens suffered heavy mortality, but there was no effect on foraging queens. The following year, we found no effect of clipping on queen survival or establishment. Clipping did not reduce overall worker survival but reduced survivorship for those in the smallest size quartile.

Implications for insect conservation Our findings suggest tarsal clipping does not have consistent negative effects on individual survival. However, our results varied with queen behavioral state, year, and worker size, suggesting differences within and among species and interactions with landscape stressors warrant further study. In the interim, we recommend researchers and conservationists minimize the use of tarsal clipping for sensitive species, populations, or small workers except in cases of exceptional scientific need.
\end{abstract}

Keywords Non-lethal sampling $\cdot$ Bombus vosnesenskii $\cdot$ Conservation genetics $\cdot$ Bumble bee rearing

\section{Introduction}

Widespread bumble bee declines have prompted a dramatic increase in the research output of laboratories and agencies focused on bumble bee monitoring and species recovery (Goulson et al. 2008; Cameron and Sadd 2020). This research effort has developed a variety of useful methods to study bumble bee individuals, colonies, and populations. As for any wild species, the impacts associated with methods used to study bumble bees must be quantified so that they

John M. Mola

jmola@usgs.gov

1 U.S. Geological Survey, Fort Collins Science Center, Fort Collins, CO, USA

2 Graduate Group in Ecology, University of California Davis, Davis, CA, USA

3 Department of Entomology, University of California Davis, Davis, CA, USA can be incorporated into analysis and minimized or avoided if deemed too harmful. A notable example is the recent listing of the federally endangered Rusty Patched Bumble bee in the United States (U.S. Fish and Wildlife Service 2017), which has motivated discussion on the safety of non-lethal sampling methods for studies of remaining populations.

Non-lethal genetic sampling of bumble bees primarily involves taking "tarsal clips" from individuals in the field (Holehouse et al. 2003). Tarsal clipping removes the distal tarsal segments of one mid-leg (Fig. 1a). The procedure yields enough genetic material to reliably extract DNA for microsatellite or other analyses, purportedly without negatively affecting individual or colony survivorship or success. The information gathered from tarsal clipping has been crucial to our understanding of bumble bees. Tarsal clips have been used to determine colony abundance, foraging and dispersal distance, and population connectivity (reviewed in Mola and Williams 2019), all of which cannot be readily estimated from transect 


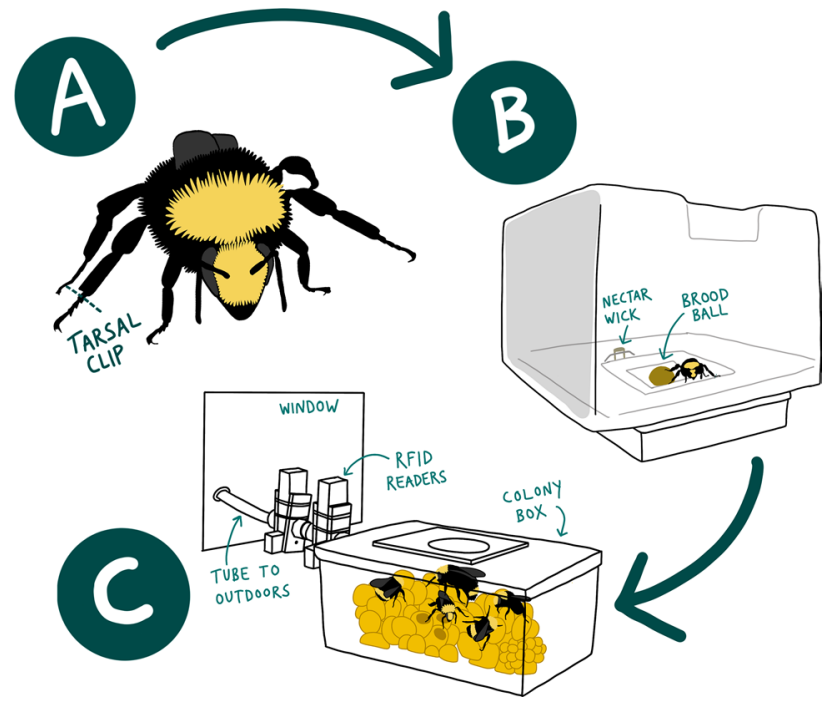

Fig. 1 Diagram of tarsal clipping, rearing boxes, and general study procedure for Bombus vosnesenskii. a Queens are clipped in the field below the tibial spurs. b In the laboratory, queens are placed into boxes with a brood ball consisting of pollen and nectar. They are also provisioned with a nectar wick that pulls a sugar solution from a reservoir below. c Mature colonies are moved into larger boxes to allow foraging outdoors through an exit tube affixed with RFID readers to track directionality. Artwork by C. Stuligross

counts of workers. To date, only one study has evaluated the potential side effects of tarsal clipping (Holehouse et al. 2003), focusing on workers of a common, commercially available species (Bombus terrestris). However, tarsal clipping has been extensively used on both workers and queens of various species (Examples with workers: Darvill et al. 2006, Charman et al. 2010, Redhead et al. 2016; Examples with queens: Lepais et al. 2010, Carvell et al. 2017, Mola et al. 2020). We must further evaluate this method to determine its risk for sensitive species, especially as potential negative effects of tarsal clipping on survivorship will have downstream effects on the estimation of demographic measures.

To better understand the potential side effects of tarsal clipping on wild bumble bees, we collected wild bumble bee queens of Bombus vosnesenskii (Radoszkowski 1862). We took tarsal clippings of approximately half of these queens and then of a subset of worker offspring of these queens. We investigated how tarsal clipping affected queen survivorship, queen nest establishment, and worker survival. We expected queens would be negatively impacted by tarsal clipping due, in part, to the demands of colony establishment. Alternatively, queens may endure tarsal clipping more readily than workers due to queen's large size. Similarly, we expected smaller workers to be more susceptible to negative effects of tarsal clipping.
Table 1 Sample sizes of queens (Bombus vosnesenskii) across location, behavior, and tarsal clipping treatment

\begin{tabular}{llccc}
\hline Year & Behavior & $\begin{array}{l}\text { Number } \\
\text { clipped }\end{array}$ & $\begin{array}{l}\text { Number } \\
\text { unclipped }\end{array}$ & Total \\
\hline 2018 & Foraging & 22 & 22 & 44 \\
& Nest searching & 6 & 5 & 11 \\
2019 & Foraging & 9 & 8 & 17 \\
& Nest searching & 21 & 21 & 42 \\
Totals & & 58 & 56 & 114 \\
\hline
\end{tabular}

\section{Methods}

\section{Field collections}

For the initial phase of this study, we collected queens of B. vosnesenskii in Monterey, CA, USA (Monterey, CA; $36^{\circ} 39^{\prime} 37.5^{\prime \prime} \mathrm{N} 121^{\circ} 48^{\prime} 38.5^{\prime \prime} \mathrm{W}$ ) in 2018 . After receiving alarming results, albeit with low sample sizes (Table 1), we repeated our collections at the University of California McLaughlin Natural Reserve (Lower Lake, CA; $\left.38^{\circ} 52^{\prime} 25.7^{\prime \prime} \mathrm{N}, 122^{\circ} 25^{\prime} 56.2^{\prime \prime} \mathrm{W}\right)$ in 2018 and again in 2019 in Monterey. We collected queens from each cohort with aerial nets during a single visit to the field site. We categorized queens according to their spring behavioral state during capture as either nest searching (moving in a characteristic nest searching pattern low to the ground investigating potential nest cavities; Svensson et al. 2000) or foraging. Upon capture, we placed queens in a cooler to temporarily immobilize them. In the field, we removed each queen in turn and took a tarsal clip from approximately half of them (Table 1) following the methods of Holehouse et al. (2003). For unclipped queens, we removed them from the cooler and placed forceps on their tarsus to simulate a similar amount of time out of the cooler and any effects of handling. These methods are all standard practice in the procurement of genetic samples from wild bumble bees. All queens were returned to the cooler and transported back to the rearing facility at the University of California Harry J. Laidlaw Jr. Honey Bee Biology Facility. There they were immediately placed into nesting boxes.

\section{Queen rearing, survivorship, and nest establishment}

We reared queens using common laboratory rearing techniques in brood boxes (BioBest Group, Westerlo, Belgium). We provided each queen with an initial $1.5 \mathrm{~g}$ brood ball consisting of a mix of honey bee-collected pollen (Koppert Biological Systems, Howell, Michigan) mixed with a solution of 1:1 distilled water:Pro-Sweet $(\sim 77 \%$ 
sugar solids, Mann Lake, Woodland, CA) and ad libitum access to 1:1 water Pro-Sweet solution supplied by a wick feeder (Fig. 1b). We monitored queens every 1-2 days and replenished their nectar and pollen as necessary.

During each monitoring event, we checked each queen box for mortality or the presence of workers after brood initiation. We followed consistent monitoring and feeding protocols for all queens for 25 days. After 25 days, we either culled queens if alive but showing no signs of broodiness (i.e. not incubating egg masses in the brood ball) or transferred them into full-size colony boxes. To determine if clipping negatively affected queens, we conducted a Kaplan-Meier survivorship analysis using the R package survminer (Version 0.4.6; Kassambara et al. 2019). We fit a survival curve with the number of days a queen was alive as a function of clipping treatment, behavioral state (foraging or nest searching), and their interaction. Data were right censored after 25 days. We conducted this analysis for queens in 2018, 2019, and for all queens pooled across years. To determine if clipping negatively affected queens' ability to produce workers, we used contingency tests of whether the number of queens that produced at least one offspring depended on clipping treatment. For this analysis we only included queens who survived at least 25 days given that dead queens could not produce workers.

\section{Worker survivorship}

We retained a subset of five large colonies ( $>50$ workers) from which to investigate the effects of tarsal clipping on worker survivorship. We transferred these colonies to larger colony boxes and connected their entrances via tubes to ports in the widows of the laboratory building (Fig. 1c). This allowed workers to forage freely by leaving the laboratory building via the entrance/exit tube. We monitored worker activity at this entrance using a 2-reader radio-frequency identification (RFID) system (Kerr et al. 2019; Nunes-Silva et al. 2019). We randomly selected 130 workers, measured their body size (intertegular distance; ITD), and affixed an RFID chip (mic3-TAGv, 64-bit RO, iID2000 13.56 MHz system, $1.9 \mathrm{~mm} \times 1.6 \mathrm{~mm} \times 0.5 \mathrm{~mm}$, Microsensys, Erfurt, Germany) to the thorax of each bee under brief $\mathrm{CO}_{2}$ anaesthetization. We took tarsal clippings from every other worker as we pulled them from their colony for marking. Workers were then returned to their colony and allowed to forage. To estimate survival of tagged workers, we calculated detection days as the day an individual was tagged until the last day it was sensed by the RFID reader. Although this may not fully cover the range of survival since it could be that clipping reduces foraging and therefore a lack of detection rather than mortality, it should represent an unbiased estimate of their total activity across all individuals and in either event would represent a loss of forager contribution to the colony. To determine whether body size affected the impact of clipping or survival, we categorized worker body size into four quartiles thus creating eight groups (clipped/unclipped $\times$ each quartile). We conducted survivorship analysis for up to 30 days by generating Cox Proportional Hazard Ratios with detection days as the response and the eight worker groups as the predictor using the coxph function within the package survival (Version 3.1.12; Therneau and Lumley 2015).

All analyses were conducted in R version 4.0.0 (R Core Team 2020).

\section{Results}

\section{Queen survival}

Tarsal clipping did not affect overall queen survival (Fig. 2a, $\chi^{2}=0.1, \mathrm{df}=1, \mathrm{~N}=114, \mathrm{P}=0.7$ ) or the interaction between clipping and behavioral state (Fig. $2 b, \chi^{2}=3$, $\mathrm{df}=3, \mathrm{~N}=114, \mathrm{P}=0.4$ ). However, there was a significant year effect with queens in 2019 having higher overall rates of mortality (Cox Proportional Hazard Ratio $=2.3$, 95\% $\mathrm{CI}=1.10-4.9, \mathrm{P}=0.027)$. Considering each year separately, we found a significant negative effect of clipping on nest searching queens in the first year (2018; Fig. 2c, $\chi^{2}=16.8$, $\mathrm{df}=3, \mathrm{~N}=55, \mathrm{P}<0.001)$, but not in the second year (2019; Fig. $2 d, \chi^{2}=2.9, d f=3, N=59, P=0.4$ ).

\section{Nest establishment}

Of the 114 queens captured, only 30 ever produced at least one worker (Table 2), due in part to a large second-day mortality of queens in 2019. Of the queens that survived, clipped queens were slightly less likely to produce a worker (37\% versus $47 \%$ ), but this effect was not statistically significant $\left(\chi^{2}=0.38, \mathrm{df}=1, \mathrm{~N}=71, \mathrm{P}=0.54\right)$.

\section{Worker survival}

Overall worker detectability was not affected by clipping $\left(\chi^{2}=1, \mathrm{df}=1, \mathrm{~N}=130, \mathrm{P}=0.3\right)$. However, clipped workers in the smallest quartile were less likely to be detected than unclipped workers of that, or any other, size class (Fig. 3).

\section{Discussion}

\section{General discussion}

Tarsal clipping stands as a reliable method for sampling genetic material from wild bumble bees. However, our inconsistent results suggest the need for further study among more species and different ecological contexts to more 

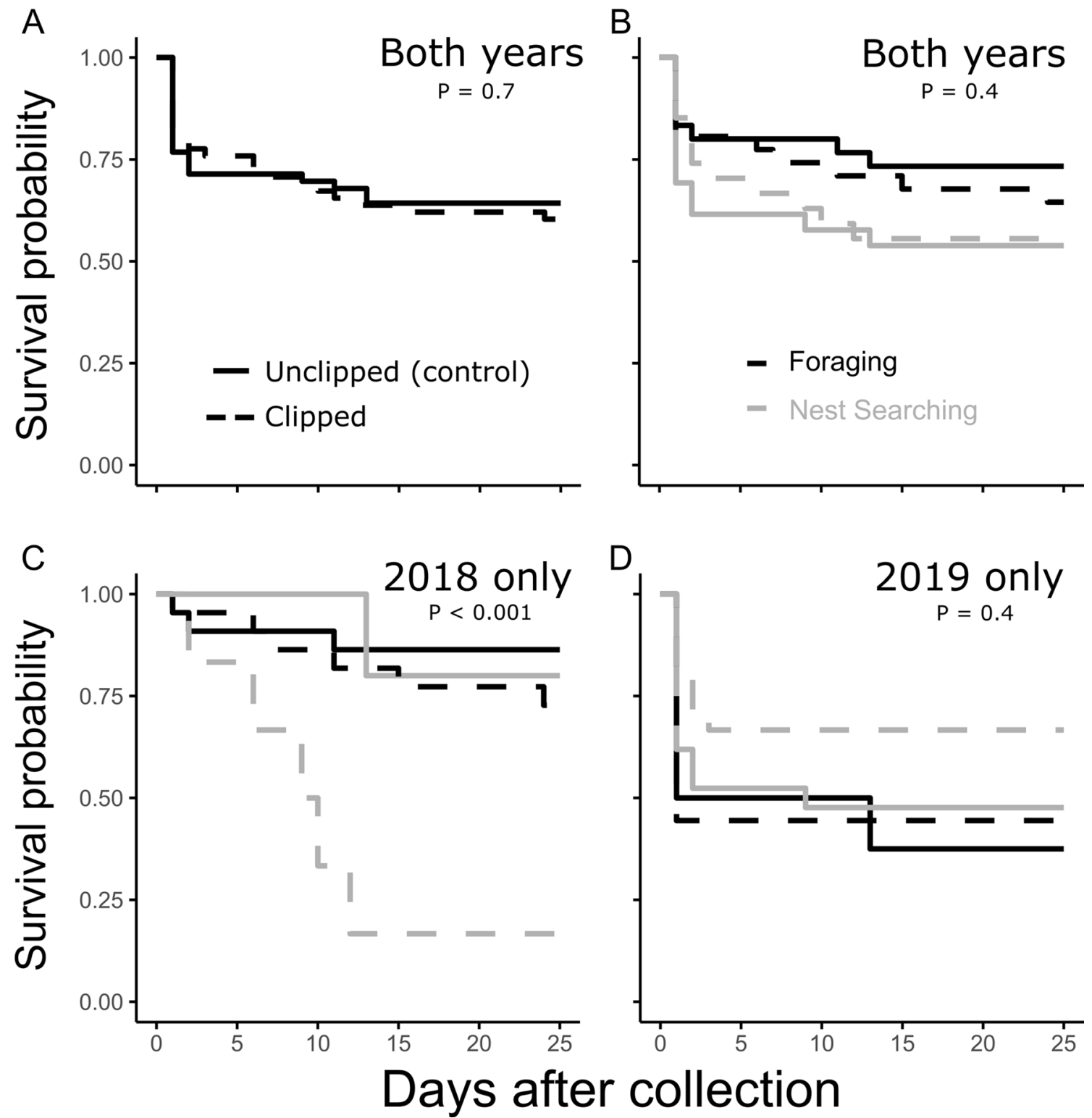

Fig. 2 Survival curves of Bombus vosnesenskii for collections of a all queens whose tarsi were clipped (dashed lines) and unclipped (solid lines), $\mathbf{b}$ all queens clipped and unclipped categorized by behavior at time of collection as either foraging (black) or nest searching (grey), c the 2018 cohort and d the 2019 cohort. Clipping had no signifi-

Table 2 The production of workers by queen (Bombus vosnesenskii) across both years of the study (2018-2019)

\begin{tabular}{llll}
\hline Clipped? & $\mathrm{N}$ & $\begin{array}{l}\text { Number of queens who } \\
\text { survived }>25 \text { days }(\%)\end{array}$ & $\begin{array}{l}\text { Number of surviving queens } \\
\text { who produced } \geq 1 \text { worker }(\%)\end{array}$ \\
\hline Yes & 58 & $35(60.3 \%)$ & $13(37.1 \%)$ \\
No & 56 & $36(64.2 \%)$ & $17(47.2 \%)$ \\
\hline
\end{tabular}

cant effect on survival except in 2018, when clipped, nest searching queens (gray, dashed line, c had lower survival probability than all other treatments. P-values generated from log-rank test of KaplanMeier survival curves

accurately understand the potential negative side effects. Clipping did not affect individual survival generally, but we observed high mortality in nest searching queens one year (Fig. 2) and in smaller workers (Fig. 3), suggesting greater scrutiny of the effects of tarsal clipping is warranted within the bumble bee conservation community. For instance, if tarsal clipping can sometimes have large negative effects, we must determine how to minimize those effects, whether alternative methods may be available (e.g. extraction of DNA from frass), and when the risk of the methodology is worth the information it provides (e.g. determining 


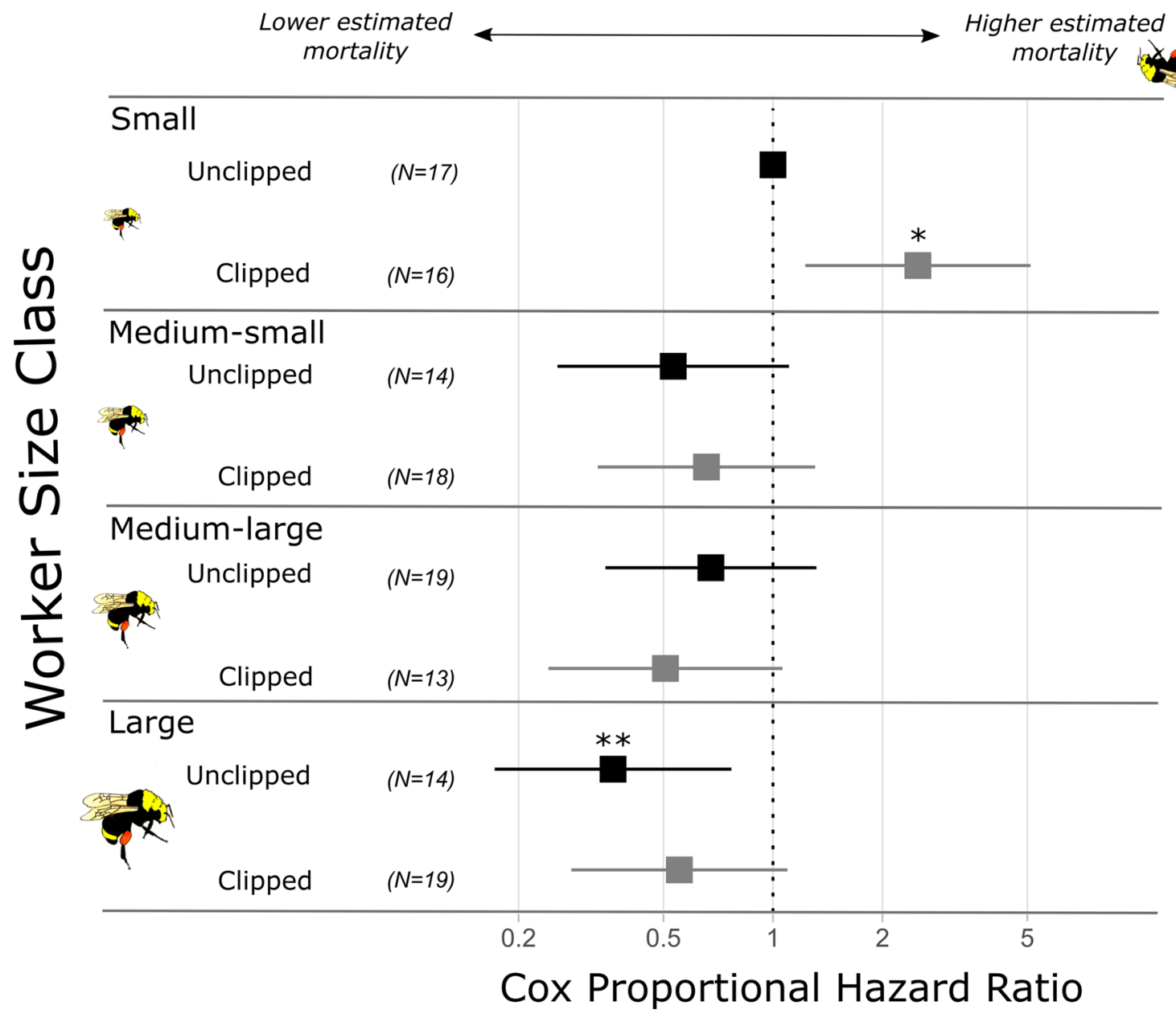

Fig. 3 Cox proportional hazard ratio of worker detection days across size classes for Bombus vosnesenskii. Values (hazard ratio $\pm 95 \%$ $\mathrm{CI}$ ) are referenced against the smallest class of unclipped workers as measured by intertegular distance (ITD) and organized from smallest (top) to largest (bottom) size class. Clipped workers within the smallest class of ITD were detected less frequently than other size

inbreeding within populations prior to ex situ management actions such as genetic augmentations or reintroductions; Smith et al. 2020). Additionally, researchers seeking to use tarsal clipping to estimate levels of lineage survival in the field may want to consider how to adjust estimates to offset effects of lethality.

We caution against extrapolating our results to full-scale skepticism about tarsal clipping and instead present them to spur more careful discussion and targeted research within the conservation community. We did not observe negative effects of clipping when we repeated our study in 2019, and the original cohort (2018) that experienced negative effects was a small sample of nest searching queens (Table 1). Nevertheless, we should consider what deleterious effects on nest searching queens might mean within the context of bumble bee behavioral state. Mortality among nest searching or clipping classes. Unclipped workers of the largest size class were detected more frequently than any other category. Size class cutoffs are quartiles representing small (below 25th percentile), mediumsmall (50th), medium-large (75th), and large (100th) sized workers. Significance derived from Wald statistic shown as $* \mathrm{P}<0.05$ and $* * \mathrm{P}<0.01$

queens exposed to clipping (i.e. mechanical disturbance) may have simply revealed weakness among these individuals, and our manipulation sped along their demise. Thus, it is possible that tarsal clipping may pose just enough additional stress to cause mortality in already stressed early-stage bees but is not sufficiently stressful to negatively affect more robust individuals. We noted that in 2018, the temperature of our rearing facility fluctuated greatly as we learned to control the conditions within the room. The interaction between a fluctuating temperature and the clipping treatment could have triggered mortality. In 2019, we stabilized temperatures in the rearing room. Targeted experimentation on tarsal clipping and environmental disturbance like heat waves, pesticide exposure, and pollen collecting would reveal these potential effects and more appropriately simulate conditions queens experience within field settings. It is possible that 
the lethality of tarsal clipping observed in 2018 is due to the combination of clipping and temporary cold storage of queens while transporting them to the laboratory. However, because we did not observe this pattern in 2019 and cold transport of queens is well established in rearing protocols (Malfi et al. 2019), this seems unlikely. Additionally, queens reared in the laboratory do not need to forage and are not subjected to the same risks or task sets as individuals in the wild, so any interactions between clipping and experimental conditions are likely smaller than the stressors encountered by wild queens. Future studies using mark-recapture of wild nesting queens to determine if clipped queens are detected less often than unclipped queens may more appropriately approximate these stressors.

Holehouse et al. (2003) stated that clipping had no negative effect on the size of workers produced after treatment but did not look at the effects of clipping on individuals across a range of body sizes. We observed reduced survival of clipped workers of the smallest size class (Fig. 3). Given that sampling of wild-foraging workers rarely results in more than a few individuals per colony being collected, maybe even complete mortality would be of minimal concern. However, colonies of many species appear to produce smaller workers early in the season (Knee and Medler 1965; Shpigler et al. 2013), and early-season foraging success is particularly important for colony success (Malfi et al. 2019). Thus, losses of even a single forager could have negative demographic consequences. Losses of larger workers would occur when colonies are also larger, thus reducing negative effects on colonies and populations. Our results suggest that researchers should avoid conducting tarsal clipping on early season workers of sensitive species or declining populations without a cost-benefit analysis comparing potential harm against research benefit.

\section{Conclusion}

We hope our findings will lead to further, careful study of the effects of tarsal clipping on multiple species, castes, and ontogenetic phases of the bumble bee life cycle and other taxa. In addition, molecular methods and DNA extraction procedures have advanced since Holehouse et al.'s (2003) important methodological study, so methods like frass samples should be revisited. For common species such as the one investigated here, and populations considered stable, there is no clear reason to reduce the use of tarsal clipping. Instead, our results suggest caution and the need for further research on sensitive species. In the interim, we suggest best practices dictate we should avoid tarsal clipping of queens and earlyseason or small workers of species in decline.

Acknowledgements We would like to thank Tamara Smith and Ian Pearse for comments on a draft of this manuscript; the UC Reserve
System for allowing us to collect samples at McLaughlin Reserve; attendees of the Rusty Patched Bumble bee ex situ management meeting for encouraging us to publish our results. We would also like to acknowledge the bees who graciously lent a foot. Any use of trade, firm, or product names is for descriptive purposes only and does not imply endorsement by the U.S. Government.

Funding JMM was supported by National Science Foundation Graduate Research Fellowship (NSF GRF) Award 1049702. CS was supported by NSF GRF. MLP was partially supported by a Department of Defense National Defense Science and Engineering Graduate Fellowship. DR was supported by a UC Davis Eugene Cota-Robles Fellowship. The work was also supported by NSF DEB 1354022 to NMW.

Data availability Data is available on Dryad digital repository at https ://doi.org/10.25338/B8CS63.

\section{Compliance with ethical standards}

Conflict of interest The author declares that they have no conflict of interest to disclose.

Open Access This article is licensed under a Creative Commons Attribution 4.0 International License, which permits use, sharing, adaptation, distribution and reproduction in any medium or format, as long as you give appropriate credit to the original author(s) and the source, provide a link to the Creative Commons licence, and indicate if changes were made. The images or other third party material in this article are included in the article's Creative Commons licence, unless indicated otherwise in a credit line to the material. If material is not included in the article's Creative Commons licence and your intended use is not permitted by statutory regulation or exceeds the permitted use, you will need to obtain permission directly from the copyright holder. To view a copy of this licence, visit http://creativecommons.org/licenses/by/4.0/.

\section{References}

Cameron SA, Sadd BM (2020) Global trends in bumble bee health. Annu Rev Entomol 65:209-232

Carvell C, Bourke AFG, Dreier S, Freeman SN, Hulmes S, Jordan WC, Redhead JW, Sumner S, Wang J, Heard MS (2017) Bumblebee family lineage survival is enhanced in high-quality landscapes. Nature 543:547

Charman TG, Sears J, Green RE, Bourke AFG (2010) Conservation genetics, foraging distance and nest density of the scarce great yellow bumblebee (Bombus distinguendus): conservation genetics of Bombus distinguendus. Mol Ecol 19:2661-2674

Darvill B, Ellis JS, Lye GC, Goulson D (2006) Population structure and inbreeding in a rare and declining bumblebee, Bombus muscorum (Hymenoptera: Apidae): inbreeding in a rare bumble bee. Mol Ecol 15:601-611

Goulson D, Lye GC, Darvill B (2008) Decline and conservation of bumble bees. Annu Rev Entomol 53:191-208

Holehouse KA, Hammond RL, Bourke AFG (2003) Non-lethal sampling of DNA from bumble bees for conservation genetics. Insectes Soc 50:277-285

Kassambara, A., M. Kosinski, and P. Biecek. 2019. survminer: drawing survival curves using'ggplot 2'.

Kerr NZ, Crone EE, Williams NM (2019) Integrating vital rates explains optimal worker size for resource return by bumblebee workers. Funct Ecol 33:467-478 
Knee WJ, Medler JT (1965) The seasonal size increase of bumblebee workers (Hymenoptera: Bombus). Can Entomol 97:1149-1155

Lepais O, Darvill B, O'Connor S, Osborne JL, Sanderson RA, Cussans J, Goffe L, Goulson D (2010) Estimation of bumblebee queen dispersal distances using sibship reconstruction method: estimation of bumblebee queen dispersal. Mol Ecol 19:819-831

Malfi RL, Crone E, Williams N (2019) Demographic benefits of early season resources for bumble bee (B. vosnesenskii) colonies. Oecologia 191:377-388

Mola JM, Miller MR, O'Rourke SM, Williams NM (2020) Wildfire reveals transient changes to individual traits and population responses of a native bumble bee Bombus vosnesenskii. J Anim Ecol. https://doi.org/10.1111/1365-2656.13244

Mola JM, Williams NM (2019) A review of methods for the study of bumble bee movement. Apidologie 50:497-514

Nunes-Silva P, Hrncir M, Guimarães JTF, Arruda H, Costa L, Pessin G, Siqueira JO, de Souza P, Imperatriz-Fonseca VL (2019) Applications of RFID technology on the study of bees. Insectes Soc 66:15-24

R Core Team (2020) R: a language and environment for statistical computing. R Foundation for Statistical Computing, Vienna

Redhead JW, Dreier S, Bourke AF, Heard MS, Jordan WC, Sumner S, Wang J, Carvell C (2016) Effects of habitat composition and landscape structure on worker foraging distances of five bumblebee species. Ecol Appl 26:726-739
Shpigler H, Tamarkin M, Gruber Y, Poleg M, Siegel AJ, Bloch G (2013) Social influences on body size and developmental time in the bumblebee Bombus terrestris. Behav Ecol Sociobiol 67:1601-1612

Smith TA, Strange JP, Evans EC, Sadd BM, Steiner JC, Mola JM, Traylor-Holzer K (2020) Rusty patched bumble bee ex situ assessment and planning workshop: final report. IUCN SSC, Apple Valley, MN, p 85

Svensson B, Lagerlöf J, Svensson BG (2000) Habitat preferences of nest-seeking bumble bees (Hymenoptera: Apidae) in an agricultural landscape. Agr Ecosyst Environ 77:247-255

Therneau TM, Lumley T (2015) Package 'survival.' R Top Doc 128:112

U.S. Fish and Wildlife Service (2017) Endangered and threatened wildlife and plants; endangered species status for rusty patched bumble bee. Federal register 82 FR 3186. U.S. Fish and Wildlife Service, Washington, DC

Publisher's Note Springer Nature remains neutral with regard to jurisdictional claims in published maps and institutional affiliations. 